\title{
LAS ORGANIZACIONES DESDE LAS CIENCIAS DE LA COMPLEJIDAD: UNA APROXIMACIÓN DESDE LA TERMODINÁMICA DEL NO-EQUILIBRIO
}

Adolfo Carbal-Herrera Universidad de Cartagena Colombia

Yury Marcela Guevara-Huertas

Universidad de Cartagena Colombia

Jaime Yasser Ochoa-Uparela Universidad de Cartagena Colombia 
Panorama Económico, Vol. 25 - No. 1 (Enero - Marzo de 2017), pp. 75-94

Adolfo Carbal-Herrera

Yury Marcela Guevara-Huertas

Jaime Yasser Ochoa-Uparela

Las Organizaciones desde las ciencias de la Complejidad: Una aproximación desde la termodinámica del no-equilibrio

\title{
Resumen
}

Este trabajo se plantea una concepción de las organizaciones desde la perspectiva de las ciencias de la complejidad, más precisamente, la termodinámica del no-equilibrio, cuyos desarrollos permiten comprender a las organizaciones como sistemas complejos, o alejados del equilibrio. Este cambio de perspectiva se ve motivado por los límites impuestos en el marco epistemológico donde reposan los cimientos de las ciencias de la gestión, es decir, aquellos que emergieron en la modernidad. El trabajo se basa en una revisión de literatura que contempla la ciencia de la complejidad, termodinámica del no-equilibrio y teoría organizacional, en bases de datos comprensivas como Scopus, ademas de Redalyc y Dialnet, y en bases de datos especializados. Se presentan los principales avances en el campo de la complejidad, como alternativas para comprender los fenómenos y entornos en los que se desenvuelven las organizaciones.

Palabras clave: Organizaciones, ciencias de la complejidad, termodinámica, sistemas complejos, teoría organizacional.

Clasificación JEL: D29, M10, M19

\section{Organizations from the Complexity sciences: an approach of non-equilibrium termodynamics}

\begin{abstract}
This research presents a revision from the perspective of complexity sciences, more precisely, non equilibrium termodynamics of the functioning of organizations. This perspective considers organizatons as complex systems that are far from equilibrium. This is motivated by the imposed limits of the basis of modern managament sciences. An extensive literature review is presented that considers complexity sciences, non-equilibrium termodynamics, and organizational theory in comprehensive scientific databases such as Scopus, iberoamerican databases (Redalyc and Dialnet), and specialized ones. The main results highlight the recent developments in the complexity field as alternative to understand the organizations' environment and phenomena.

Keywords: Organizations, Complexity Sciences, Termodynamics, Complex Systems, Management Theory. JEL Classification: D29, M10, M19
\end{abstract}

\section{Organisations issues des sciences de la complexité: une approche de la thermodynamique hors équilibre}

\section{Résumé}

Cette recherche présente une révision du point de vue des sciences de la complexité, plus précisément de la thermodynamique non équilibrée du fonctionnement des organisations. Cette perspective considère les organisations comme des systèmes complexes, loin de l'équilibre. Ceci est motivé par les limites imposées de la base des sciences modernes du management. Une revue de littérature approfondie est présentée qui prend en compte les sciences de la complexité, la thermodynamique hors équilibre et la théorie de l'organisation dans des bases de données scientifiques complètes telles que Scopus, des bases de données ibéroaméricaines (Redalyc et Dialnet) et des bases de données spécialisées. Les principaux résultats mettent en évidence les développements récents dans le domaine de la complexité comme alternative pour comprendre l'environnement et les phénomènes des organisations.

Mots-clés: Organisations, Sciences de la complexité, Termodynamique, Systèmes complexes, Théorie de la gestion.

Nomenclature JEL: D29, M10, M19 


\section{Las Organizaciones desde las ciencias de la Complejidad: Una aproximación desde la termodinámica del no-equilibrio}

INFORMACIÓN DEL ARTÍCULO

Recepción de artículo: 27/07/2016

Concepto de evaluación: 25/09/2016

Aceptación de artículo: 22/10/2016
Adolfo Carbal-Herrera* Universidad de Cartagena, Colombia

Yury Marcela Guevara-Huertas Universidad de Cartagena, Colombia Jaime Yasser Ochoa-Uparela Universidad de Cartagena, Colombia

\section{INTRODUCCIÓN}

La teoría organizacional, cuya historia retrata más de un siglo de evolución, nace en un contexto económico marcado por el mecanicismo y el determinismo de la ciencia moderna, adoptado por las ciencias sociales a causa de sus exitosas premisas, como el progreso indefinido y la elevación de la racionalidad como dogma regente para el entendimiento de la naturaleza y el universo (Capra, 1998; Chanlat, 2011). En este sentido, la modernidad es cuna de grandes descubrimientos y avances tanto en el campo de las ciencias naturales, como sociales y humanas. La necesidad de revisar la tradición epistemológica que ha regido a la teoría organizacional en el marco de las nuevas dinámicas empresariales y tendencias mundiales, proviene de las concepciones mecanicistas heredadas de las ciencias duras a las blandas, que hacen menos acertado al sustrato teórico mediante el cual se ha interpretado a las organizaciones (Aktouf, 2009; Morgan, 1998).

Algunos de los primeros limitantes que se observan en el dogma mecanicista cartesiano, reduccionista o bien determinista newtoniano, se hallan en el seno de las mismas ciencias naturales, más precisamente, en la rama de la física conocida como termodinámica (estudia la mecánica del calor y la energía) (Prigogine \& Stengers, 1994). A través de esta derivación de la física nace la necesidad de abordar los estados de equilibrio en sistemas termodinámicos, además, subyacen principios tan importantes como "la ley de la conservación de la energía", y "la ley de la entropía"; desarrollos teóricos que generaron repercusiones en su contexto histórico, así como en otros campos del saber, principalmente gracias a la termodinámica del no-equilibrio o sistemas alejados

\footnotetext{
* Autor para correspondencia

Correos electrónicos: acarbalh@unicartagena.edu.co*,yurimar95@hotmail.com, jaime-ou3@hotmail.com
} 
del equilibrio (Prigogine \& Stengers, 1994; Sandino \& Montiel, 2012).

A principios del siglo $\mathrm{XX}$, este nuevo marco de ideas se ve impulsado por la revolución cuántica en la física, con grandes exponentes como Einstein, Bohr, Schrödinger, Planck, Heisenberg, entre otros, cuyos aportes demuestran la imposibilidad de mantener vigente el dogma mecanicista, dado que sus principios no se cumplen a cabalidad a la luz de las diferencias inherentes en las leyes que rigen al mundo microscópico y macroscópico (Maldonado, 2011). Este contexto dio paso al desarrollo de las ciencias de la complejidad, aquellas caracterizadas por dejar de lado la necesidad de predecir; abarcando entonces la incertidumbre e indeterminación, así como la contradicción y la totalidad.

En consecuencia, los postulados básicos de la teoría organizacional hacia finales del siglo XIX e inicios del XX, el dogma mecanicista impregnado en las ciencias sociales, y luego extrapolado a las ciencias de la gestión, se comienzan a resquebrajar a la luz de la revolución en las ciencias naturales (Chanlat, 2011; Goméz-Cruz \& Maldonado, 2011; Naredo, 2003). En este orden de ideas, se evidencia el surgimiento de nuevas concepciones que pretenden abordar los desarrollos teóricos en materia de gestión a través de los lentes de la complejidad. En este sentido, la propuesta de este texto tiene por objeto impulsar el entendimiento de los fenómenos y contextos organizacionales desde las ciencias de la complejidad, en particular, desde la visión de la termodinámica del noequilibrio, permitiendo la comprensión de las organizaciones desde dichos sistemas.

El documento se estructura en cuatro (4) acápites así: el primero, aborda las ideas fundamentales que subyacen en el pensamiento moderno $y$ que han permeado a las ciencias sociales y humanas, entre ellas, las de gestión; el segundo, presenta los primeros indicios de complejidad en el seno de la termodinámica clásica; el tercero, esboza algunos planteamientos generales sobre la termodinámica del no-equilibrio o de los sistemas alejados del equilibrio en el marco de las ciencias de la complejidad; y el último, muestra sucintamente un recuento de los principales postulados de la teoría organizacional, a su vez, que plantea la comprensión de la organización desde los planteamientos alternativos de la termodinámica del no-equilibrio.

\section{Metodología}

El texto corresponde a una revisión sistemática de literatura sobre temas tales como: ciencias de la complejidad, termodinámica del no-equilibrio y teoría organizacional, en bases de datos como: Scopus, Redalyc y Dialnet, y en buscadores especializados como Google Académico; haciendo uso de descriptores como: "ciencias de la complejidad", "termodinámica del no-equilibrio", "teoría organizacional", y ecuaciones de búsqueda como "organizaciones y ciencias de la complejidad". Los textos seleccionados se agruparon en tres categorías de análisis: ciencias mecanicistas, termodinámica del no equilibrio y teoría organizacional desde una concepción compleja. Se escogieron los textos de mayor relevancia de acuerdo con las categorías propuestas, posteriormente se elaboraron fichas de lectura, se hizo uso del gestor bibliográfico Medeley para la organización de la literatura revisada y el almacenamiento de las notas bibliográficas elaboradas por cada texto, a partir de las cuales se estructuró la presente reflexión. 


\section{Resultados y discusión}

\section{El camino hacia el progreso y la sublevación de la racionalidad}

La ciencia, entendida para los griegos como búsqueda de la verdad, ha sido de importancia histórica para el desarrollo del ser humano. Las primeras disertaciones planteadas sobre la naturaleza del conocimiento, los astros y el universo, datan de la época antigua donde la realidad era concebida como un todo armonioso, el cual funcionaba a través de un orden establecido o cósmico, cuyas excepciones a la regla se entendían como los desequilibrios de aquella unión (Capra, 1998; Schrödinger, 1997). Esta visión que reinó durante toda la antigüedad, y que indirectamente fue acogida en el seno del medioevo a través de la imagen de Dios como ente regente de aquella armonía, experimentó una profunda transformación producto del despertar del hombre, y más precisamente de su razón como nueva forma de comprender los fenómenos que se vislumbraban ante él, como desconocidos y conformemente aceptados a causa de las explicaciones divinas (Mumford, 1998; Najmanovich, 2005).

Los cambios acaecidos a razón del auge de la modernidad, con la revolución científica de los siglos XVI y XVII, generaron una visión radicalmente distinta de la realidad; donde las ciencias naturales, en aquella época denominada filosofía natural, desentrañaron grandes misterios y produjeron grandes avances para la civilización moderna que se gestaba (Capra, 1992). Uno de los precursores de dicha transformación social, cultural y por sobre todo científica, fue Nicolás Copérnico. "La revolución copernicana fue una revolución en el campo de las ideas, una transformación del concepto del universo que tenía el hombre hasta aquel momento y de su propia relación con el mismo" (Kuhn, 1978, pág. 14). Copérnico se propuso incrementar la precisión y sencillez de la teoría astronómica vigente transfiriendo al sol muchas de las funciones que hasta entonces se atribuían a la tierra, visión del universo que fue incompatible con la de otros científicos, sin embargo, la reconciliación de dicha discrepancia fue parte importante para la construcción generalizada de lo que hoy llamamos revolución científica (Kuhn, 1978).

Por otro lado, personajes como Tycho Brahe y Johannes Kepler jugaron un papel importante al desarrollar las tablas astronómicas y de ellas formular las leyes que rigen el movimiento de los planetas (Koyré, 1979). Sin embargo, no fue hasta la llegada de Galileo Galilei quien a través del telescopio comprobó la visión copernicana, además, de impulsar la experimentación y medición, con sus estudios sobre el movimiento de los cuerpos, como formas objetivas de conocimiento científico (Koyré, 2008). Otro gran aporte fue el del inglés Francis Bacon, el cual desarrolló incipientemente la filosofía positivista, más adelante instaurada en las ciencias por Augusto Comte.

En este orden de ideas, uno de los máximos exponentesdela nueva visión dela realidad fue René Descartes, con su duda metódica, como forma de alcanzar el conocimiento verdadero. Es a este filósofo, matemático y físico francés a quien debemos la concepción del mundo fragmentado, del universo como máquina perfecta gobernada por leyes matemáticas exactas, que podía ser enteramente comprendidas. “Descartes creó el método de pensamiento analítico, consistente en desmenuzar los fenómenos complejos en partes para comprender, desde las propiedades de 
éstas, el funcionamiento del todo" (Capra, 1998, pág. 39). Por su parte, Isaac Newton, considerado uno de los más grandes genios de la historia de la ciencia, hizo sus aportes en matemática, astronomía y física, los cuales representaron la síntesis de la revolución científica; y cuya importancia es vislumbrada en el famoso epitafio escrito por Alexander Pope: la naturaleza y sus leyes yacían dormidas en la oscuridad, y Dios dijo, que sea Newton y todo fue luz (Koyré, 1979; Mumford, 1998; Prigogine \& Stengers, 1994).

Este nuevo paradigma mecanicista fundamentó sus creencias en la metáfora de la máquina, donde el comportamiento de los seres vivos, la naturaleza y el universo es semejante al funcionamiento de las máquinas, lo que permite afirmar que se puede predecir, determinar o estudiar de forma reduccionista, o fragmentando el todo en sus partes constituyentes (Capra, 1992; La Mettrie, 1987; Prigogine, 1996). La sublevación de este paradigma trajo consigo el establecimiento de algunas ideas importantes en el pensamiento colectivo, por ejemplo: el progreso indefinido y la exaltación de la racionalidad. Estas premisas se tornaron el motor de arranque para los descubrimientos y avances posteriores en ciencias naturales; y más adelante, en aquellas, que al ver dicho éxito quisieron asemejarse a las anteriores, las ciencias sociales (Naredo, 2003).

En este sentido, es necesario destacar que el siglo XVI también fue época de cambios socio-culturales en cuanto al sistema o modo de producción que regía a la sociedad. El feudalismo, paulatinamente, dio paso a las primeras manifestaciones del capitalismo, el cual impuso nuevas ideas en el comercio, así como en los aspectos económico, político y social, gracias a su rápida expansión producto del descubrimiento de américa (Fromm, 1964). De esta forma, los éxitos alcanzados en las ciencias naturales con el dogma mecanicista permearon los desarrollos en ciencias sociales y humanas, de tal forma, que el método científico planteado por la filosofía positivista jugó un papel importante como catalizador en el proceso descrito anteriormente (Marín-Idárraga, 2012; Naredo, 2003).

El economista José Manuel Naredo plantea que las primeras nociones de lo económico, entendido para la época como aritmética política (término acuñado por William Petty), se fundaron sobre la base del pensamiento mecanicista, impactando en las escuelas precursoras de la economía clásica, más precisamente, en el pensamiento de Adam Smith, David Ricardo y Thomas Malthus; los cuales impusieron la idea de cuantificación como el horizonte deseado de los economistas. Esta quimera es alcanzada con la revolución neoclásica dado que sus máximos exponentes lograron permear con las leyes matemáticas las ideas que plantearon sus antecesores, lo cual logró la cuantificación de la economía, un ejemplo de esto corresponde al principio de utilidad de Bentham. Lo que permitió perpetuar nociones como el comportamiento causal de los seres humanos, la percepción del homo economicus, y la idea de consumo como motor de satisfacción y felicidad (Naredo, 2003).

En este orden de ideas, las ciencias de la gestión no son un caso aparte en el entramado de la historia moderna. Los pilares que constituyen la base de todo el pensamiento administrativo recaen sobre las propuestas clásicas de Taylor, Fayol y, desde una perspectiva sociológica, Weber; los cuales exaltaron la labor de la racionalidad en el estudio de las organizaciones 
modernas, además de sentencias como: la división y especialización del trabajo, el análisis científico de las operaciones y fenómenos empresariales, entre otras (Aktouf, 2009; Chanlat, 2011; Marín-Idárraga, 2012). De esta forma, Gareth Morgan sustenta la presencia del pensamiento mecanicista en los cimientos de la administración moderna (Morgan, 1998).

\section{De los primeros indicios de complejidad en la termodinámica clásica}

Las ideas máximas de la doctrina mecanicista como el progreso indefinido, la racionalidad y el método científico, perduraron hasta la revolución einsteniana de principios del siglo XX (Einstein \& Infeld, 1986). Estas premisas y los adelantos en mecánica durante el siglo XVII y XVIII gestaron las primeras nociones de la termodinámica; las cuales se constituyeron fuera del universo determinista newtoniano, pero con una magnitud similar a sus leyes; así, el mecanicismo se consolidó como la ciencia del movimiento (dinámica), no obstante, la termodinámica y la biología evolutiva, que se gesta más adelante, se erigen como las ciencias de las interacciones y el cambio (Prigogine \& Stengers, 1984; Prigogine \& Stengers, 1994; Sandino \& Montiel, 2012).

En este sentido, es necesario recalcar que el avance de la técnica a finales del siglo XVIII e inicios del XIX fue el punto de partida de otros adelantos, que se consagraron a la explotación de las fuerzas naturales (Heisenberg, 1985). Este fue el contexto que propició la utilización del vapor para trabajos técnicos, lo que consecuentemente desembocó en el desarrollo de la denominada "máquina de fuego" por Savery, que estimuló a otros ingenieros y científicos a perfeccionar la misma; cometido logrado por Newcomen años más tarde con la tradicional máquina de vapor. Para mediados del siglo XVIII, dicho invento fue perfeccionado por James Watt hasta lograr su adaptación en otras áreas industriales (Torreblanca, 2013).

En el contexto histórico de inicios del siglo XIX, las necesidades productivas se incrementaron, deformatal, quelostalleres artesanales de la época se conglomeraron en incipientes industrias manufactureras; lo que eventualmente produjo el barco a vapor y la locomotora (Sandino \& Montiel, 2012). La ampliación de la ciencia fue inevitable, y la termodinámica destacó al impulsar el surgimiento de nuevas concepciones mecánicas (Turegano \& Valero, 1982). De esta manera, aquella se desarrolla en el transcurso del siglo XIX como una rama de la física que estudia el calor, energía y el equilibrio; e inicia con la obra de Sadi Carnot publicada en 1824 "Reflexiones sobre la potencia motriz del fuego y sobre las máquinas diseñadas para desarrollar dicha potencia".

Carnot fue el primero en destacar la importancia del poder transformador del fuego como fuente motora para los artefactos de la época, además, intuyó que la civilización estaba experimentando una revolución hacia la industria debido a las máquinas térmicas (Carnot, 1897; Pérez, 2007). Con este impulso, algunos científicos como Joule, Mayer, Thomson, Clausius y Boltzmann; pasaron a representar la cúspide de la termodinámica, ya no como ciencia del calor, sino de la energía (Maldonado, 2011); y formularon sus leyes o principios fundamentales: el cero, o el equilibrio térmico; el primero, la conservación de la energía; el segundo, la entropía; y el tercero, el cero absoluto (Martínez, 1996). Se resaltan el primero y el segundo, cuyos desarrollos darán pie a la ciencia de la termodinámica del no-equilibrio (Kondepudi \& Prigogine, 
2014; Maldonado, 2011; Prigogine \& Stengers, 1994).

La primera ley de la termodinámica permite comprender que en todo sistema donde haya transferencia de energía de un cuerpo a otro, aquella se transformará de acuerdo a la ley de la conservación de la energía, es decir, la energía no puede crearse ni destruirse, solo transformarse (Kondepudi \& Prigogine, 2014). En el marco termodinámico, se entenderá al ser humano como máquina energética, a la sociedad como motor, y la naturaleza en función de la energía; además, se estudiará si esta conduce o no a la entropía, cumpliendo un papel constructivo. En este contexto, surgen problemas en ciencias sociales y humanas, como los relativos a la economía y ecología en función de las fuentes de energía (Maldonado, 2011); los cuales se explican por la fundamentación mecanicista que prevalece en la economía, que desconsidera la importancia de los recursos naturales, a pesar de estar inseparablemente ligada al medio ambiente (Georgescu-Roegen, 1977; Georgescu-Roegen, 1996).

Por otro lado, la segunda ley de la termodinámica, que trata de la entropía, se refiere al proceso de disipación de energía en un sistema, en términos coloquiales, al desorden del mismo; del cual puede emerger también una situación de equilibrio (Kondepudi \& Prigogine, 2014; Maldonado, 2011). Partiendo de lo anterior, Ludwig Boltzmann descubrió cómo medir la entropía matemáticamente, y en su intento denotó que el equilibrio es el estado máximo de probabilidad. En este sentido, la termodinámica clásica muestra un universo en constante disipación, que se dirige irremediablemente hacia la "muerte térmica"; o su estado máximo de probabilidad (Durán, 2016).
En este orden de ideas, la formulación de la ley de la entropía se presenta en contradicción del universo idealizado y estable de la dinámica newtoniana, donde los procesos son reversibles y forman parte de un todo ordenado; en contraste, la entropía vislumbra un universo de procesosirreversiblescomolareglageneral, y de reversibles como simples excepciones. Es de resaltar, que en este contexto también se empieza a tener en cuenta la dimensión temporal en los fenómenos que describen sistemas entrópicos; cuya inquietud es ampliamente abordada por Prigogine en sus estudios (Prigogine, 2006; Prigogine, 1980; Prigogine, 1993; Prigogine \& Stengers, 1991).

La concepción clásica de la termodinámica abordada hasta el momento trata los sistemas aislados, y ofrece a su vez, la primera explicación sobre la complejidad presente en la naturaleza, descrita como: "...el resultado de la disipación de energía, el olvido de las condiciones iniciales, la evolución hacia el desorden, o también, la producción de orden nuevo" (Maldonado, 2011, pág. 78). Esto explica la compleja naturaleza de un universo en constante disipación, que es regido por la entropía. Sin embargo, aún surge el interrogante sobre la exhibición de vida y su evolución en este mismo universo, donde parece coexistir una flecha temporal hacia el equilibrio termodinámico y otra hacia la complejidad de la vida en sí (Maldonado, 2011).

Más adelante, empezará a cobrar importancia la interrelación con el ambiente o contexto en el que los sistemas se desenvuelven (sistemas abiertos), comprendiéndose, que estos son los preponderantes en la naturaleza, yes precisamente porque exhiben vida (Maldonado, 2011; Marin, 2014; Martínez, Ortiz, González, \& Brito, 2009). 
Así, la emergencia de esta característica en un sistema regido por la entropía, conlleva un problema que Prigogine se encargará de solucionar (Prigogine \& Stengers, 1994); y razón por la cual se introduce la noción de complejidad en el universo como el resultado de la existencia de vida en él (Maldonado, 2011).

\section{La termodinámica del no-equilibrio en el marco de las ciencias de la complejidad}

Las leyes de la termodinámica clásica se erigieron como base de una nueva ciencia cuya explicación no se ceñía a la física tradicional y su ambición; "reducir la complejidad de los fenómenos naturales a la simplicidad de los comportamientos elementales" (Prigogine \& Stengers, 1994, pág. 161). No obstante, la disociación entre paradigmas se acentuaría de forma tal que la transformación científica sería inminente en el siglo XX; dado que el siglo XIX no solo fue cuna del nacimiento $\mathrm{y}$ evolución de la termodinámica clásica, sino también, de los antecedentes a las concepciones no-lineales que serían de gran importancia para las ciencias de la complejidad, y en especial para la termodinámica del no-equilibrio (Martínez et al., 2009).

En este marco de ideas, una de las primeras visiones seminales es atribuible a Charles Darwin y sus estudios sobre el origen de las especies. La propuesta de Darwin que entrelaza dialécticamente el medio, el entorno y la adaptación; abre una brecha en la ciencia positivista, y además, representa la naturaleza de los sistemas abiertos, u organismos, comportamientos o fenómenos vivos, cuya principal característica es la complejidad creciente y alejada del equilibrio, que a su vez, tiende a auto-organizarse. Bajo este mecanismo evolutivo, la emergencia de vida, y de nuevas propiedades en el universo es posible (Bunge, 2004; Herrero, 2008; Maturana \& Varela, 1990; Mora \& Pérez, 2006); así, la no-linealidad desde el punto de vista biológico, es el resultado de la interacción entre los organismos vivos con propósitos regulatorios (Herrero, 2008).

Por otro lado, en Ludwig Boltzmann también se hallan ideas seminales, pero, desde el paradigma físico; las cuales parten de sus estudios en mecánica estadística para medir la entropía y culminan con su principio de orden. Aunque, antes de Gibbs y Boltzmann ya se había asociado la temperatura al movimiento molecular, y el calor a la energía mecánica desordenada; el aporte de esta visión radica en la interpretación microscópica del aumento de la entropía fundamentada en la probabilidad, lo que demuestra que las leyes de la termodinámica derivan de la dinámica caótica microscópica (Giorgine \& Turchetti, 2006); esto representa para los sistemas físicos, la transformación del orden al desorden como proceso irreversible (Maldonado, 2011; Marin, 2014; Martínez et al., 2009; Prigogine \& Stengers, 1994).

Siguiendo el espíritu revolucionario de Boltzmann y Darwin, a finales del siglo XIX el famoso matemático Henri Poincaré realizó importantes aportaciones a la comprensión no-lineal en su campo partiendo desde la topología, probabilidad, ecuaciones diferenciales, entre otras. Poincaré demostró que la predicción determinista solo es aplicable a algunos sistemas hamiltonianos como: oscilador armónico, el problema de los dos graves, la acción/reacción entre dos sistemas, la palanca y otros problemas mecánicos (García-Olivares, 1999). Sin mencionar, el conocido problema de los tres cuerpos de Poincaré mediante el cual, en 
términos de Prigogine, logró demostrar la imposibilidad de reducir o eliminar las interacciones; y en este mismo problema, describió por primera vez la idea del caos, de forma tal, que un sistema no-lineal, así sea uno simple podía desembocar en gran complejidad (Maldonado, 2011; Martínez et al., 2009; Prigogine \& Stengers, 1984).

En consonancia con los pioneros de la no-linealidad antes expuestos, Prigogine y Stengers (1994) resaltan la labor de Onsager a inicios del siglo XX, el cual aportó en termodinámica las "relaciones de reciprocidad" en la región cercana al equilibrio, además, afirman que aquellas fueron un punto crucial para el cambio de la termodinámica clásica a la del noequilibrio. En este sentido, se distinguen tres estadios de la termodinámica: el primero es el del equilibrio, donde los flujos de energía y las fuerzas concomitantes son todas nulas; el segundo es el de la región cercana al equilibrio, donde las fuerzas son débiles y el flujo es una función lineal de la fuerza; y el tercero de los sistemas alejados del equilibrio o no lineales, donde las características anteriores se complejizan y se torna difícil su explicación (Kondepudi \& Prigogine, 2014; Maldonado, 2011; Marin, 2014; Prigogine \& Stengers, 1994; Prigogine \& Stengers, 1984).

Todo lo anterior, deja entredicho que han existido una serie de contribuciones históricas, que paulatinamente, forjaron la visión no-lineal de la realidad; aunque no hayan sido suficiente en sus inicios para revolucionar el reduccionismo y determinismo en las ciencias. Empero, la transición entre el siglo XIX y el XX, evidentemente, fue trascendental para el cambio de concepción que se gestó en el transcurso de la centuria pasada (Martínez et al., 2009; Prigogine \& Stengers, 1994). Esta inició con las contribuciones de
Einstein y su teoría de la relatividad, la cual derrumbo finalmente, en el mismo campo de la física, la mecánica newtoniana (Einstein \& Infeld, 1986).

Asimismo, esta nueva revolución científica fue continuada con los aportes de otros grandes científicos como: Heisenberg, Bohr, Schrödinger, Planck, entre otros; que impulsaron la mecánica cuántica, y la física atomista, desarrollando un interés especial por el mundo microscópico, el cual se veía regido por leyes radicalmente distintas a las del mundo macroscópico (Capra, 1992; Capra, 1998; Gell-Mann, 1998; Heisenberg, 1985; Prigogine \& Stengers, 1994; Schrödinger, 2005). De esta manera, algunos de los exponentes de esta nueva física afirmaban que las tensiones soportadas en la época eran producto de las insuficiencias de las concepciones mecanicistas, que en consecuencia hacían tambalear los cimientos que regían la interpretación usual de sus observaciones (Bohr, 1934; Heisenberg, 1959).

En este contexto, la ciencia normal empezó a vivir una verdadera revolución científica, donde el paradigma imperante se vio insuficiente ante las nuevas concepciones; cobra relevancia el concepto Kuhniano de paradigma como una constelación de logros, valores, técnicas que son compartidos por una comunidad científica y usados para definir problemas y soluciones legítimas (Kuhn, 1962). Este fue el salto que dio la ciencia debido a la transformación de la física moderna a la contemporánea, donde nacen algunos límites como: la imposibilidad de viajar a una velocidad superior a la de la luz, el principio de incertidumbre de Heisenberg, el comportamiento (sea como onda o partícula) de un fotón, entre otras (Maldonado, 2011). 
No obstante, hay que anotar que las primeras concepciones acerca del comportamiento incierto, caótico o no-lineal en la naturaleza tienen referentes como los expuestos al inicio de este apartado. Sin embargo, a la luz de lo presentado hasta el momento, emergió un campo de estudio, que se conoce actualmente como "las ciencias de la complejidad", caracterizadas por estudiar sistemas, comportamientos y fenómenos complejos, conocidos como aquellos que exhiben incertidumbre, contradicción y totalidad; y cuyo mayor exponente es la vida (Gell-Mann, 1998; Schrödinger, 2005). Las ciencias de la complejidad son: la termodinámica del no-equilibrio (Prigogine \& Stengers, 1994); la teoría del caos (Lorenz, 1963); la geometría fractal (Mandelbrot, 1982); la teoría de las catástrofes (Thom, 1989); las ciencias de redes (Barabasi, 2002; Strogatz, 2003; Watts, 2004); y las lógicas no clásicas, donde se puede destacar la fuzzy (Zadeh, 1965).

En este marco de acción, la termodinámica del no-equilibrio se considera como uno de los campos precursores de las ciencias en mención, de hecho, Prigogine suele titular en sus obras "ciencia de la complejidad" a la termodinámica (Prigogine \& Stengers, 1994; Prigogine \& Stengers, 1984), y es precisamente, por las implicaciones y desarrollos en el área. El mérito de Prigogine, junto con Stengers recae en conciliar la ley de la entropía como la medición del desorden, disipación, o el estado de máxima probabilidad de equilibrio de un sistema, con la tendencia evolutiva y creativa de la vida, hacia estados de mayor complejidad y autoorganización (Prigogine \& Stengers, 1994).

En este sentido, uno de los mayores logros de Prigogine, que al mismo tiempo le hizo merecedor del Nobel de Química, fue la identificación de las estructuras disipativas; las cuales son formas de designar a los fenómenos o sistemas complejos, y que contribuye a entender que la disipación de energía y materia en un sistema, asociada a la pérdida de rendimiento y evolución hacia el desorden se convierte, lejos del equilibrio, en fuente de orden (Prigogine \& Stengers, 1984). En términos de Prigogine, en el equilibrio la materia es ciega, pero lejos del equilibrio, la materia ve (Prigogine, 1997); lo que conduce a la conclusión de que el orden sucede por fluctuaciones (Gell-Mann, 1998; Prigogine \& Stengers, 1994).

Por otro lado, es necesario mencionar que la identificación de estructuras disipativas de Prigogine, en los sistemas alejados del equilibrio, se complementa con otras contribuciones como: la concepción sistémica, que trata de las propiedades organizativas de los seres vivos y su interconexión, y que se atribuye a Bertalanffy, aunque planteamiento similares fueron expuestos en primera instancia por Bogdanov en su teoría denominada "Tektología" (Bertalanffy, 1989; Capra, 1998); el desarrollo del concepto de autopoiesis, como la capacidad de un sistema de auto-producirse (Maturana \& Varela, 1990); o incluso aún, la hipótesis Gaia, que plantea a la tierra como un super-organismo con funciones auto-regulatorias (Lovelock \& Margulis, 1996); entre otros adelantos.

También es importante resaltar algunos aportes de las ciencias de la complejidad como: la sensibilidad de las condiciones iniciales o el efecto mariposa dentro de la teoría del caos; la posibilidad de modelar de forma más precisa la naturaleza, así como la característica de auto-similitud en la misma dentro de la geometría fractal; la emergencia de cambios súbitos en la teoría de las catástrofes; la interconexión 
e interrelación entre los sistemas que conforman la realidad (expresada sucintamente en la teoría de los seis grados) dentro de la ciencia de redes; y la posibilidad de medir de forma más precisa y acertada la subjetividad de la realidad a través de la lógica fuzzy, como un ejemplo de las lógicas no clásicas (Goméz-Cruz \& Maldonado, 2011).

\section{Desentrañando la teoría organizacional:} los sistemas alejados del equilibrio como alternativa

Como bien se planteó al final del primer acápite, las ciencias de la gestión no fueron una excepción ante los cambios impuestos por la modernidad; la exacerbación de la racionalidad, la experimentación, medida y cálculo. Todas características de un sistema económico que floreció bajo el ala del pensamiento moderno, cuyo discurso positivista fundado en el ideal matemático de Galileo, además, de las filosofías deterministas y reduccionistas, se instauró en la sociedad como regla (Chanlat, 2011; Gómez, 2014). En este sentido, el contexto de la economía de libre mercado, donde primaba la competencia y la lógica del beneficio, fue la semilla que fecundó a la administración científica y moderna (Aktouf, 2009; Marín-Idárraga, 2012).

El ingeniero Frederic W. Taylor basó sus estudios sobre la administración, en la aplicación del método científico para incrementar la eficiencia y productividad en las industrias. Esto desembocó en la ORT (organización racional del trabajo) y sus principios para la administración científica(Álvarez, 2010; Taylor, 1919). Por otro lado, su contemporáneo Henri Fayol, conocidocomoel padredela administración moderna, fue uno de los precursores en el desarrollo de una visión anatómica (estructural) y fisiológica (funcional) de las organizaciones, además, de describir las principales funciones de las mismas, y catorce principios en procura de su correcta gestión (Fayol, 1917). Por otro lado, también se le considera como clásico al sociólogo Max Weber, cuyos aportes en cuanto a la forma de organización burocrática basada en la racionalidad, se constituye como complemento a las teorías de Taylor y Fayol (Aktouf, 2009; Morgan, 1998).

En este marco clásico de la administración, y bajo la influencia del paradigma moderno, se consolidaron ideas como: el comportamiento racional del ser humano, y la visión de "homo economicus" del mismo, que reducía al ser a una simple pieza del engranaje organizacional del cual hacía parte, y cuyo ejemplo por excelencia sería la explotación laboral de la era industrial (Chanlat, 2011; González, 2010; Gómez, 2014); también se resalta la concepción mecanicista de las organizaciones, donde estas se pueden reducir a simples elementos sin conexión aparente, y cuyo estudio fragmentado puede brindar una visión general de ella al yuxtaponer las partes y además, determinar o predecir su comportamiento (Aktouf, 2009; Le Moigne, 1997; López, 2005; Marín-Idárraga, 2012; Naredo, 2003).

En este sentido, la excesiva racionalidad producto del sistema tayloriano fue desembocando poco a poco en tratos inhumanos y explotadores hacia el trabajador (a pesar que el mismo Taylor abogara por la prosperidad de empleados y empleadores) (Álvarez, 2010; MarínIdárraga, 2006). Esto provocó que las concepciones organizacionales evolucionaran desde la visión mecanicista hacia la humanista, haciendo que los estudios de Mayo y sus acólitos ganaron importancia. Mayo, inicialmente buscaba las causas de la baja en la productividad, 
no obstante, el experimento en la Western Electric Company, denominado "efecto Hawthorne" arrojó resultados imprevistos que provocaron un cambio en su dirección; determinando el impacto de los aspectos sociales y psicológicos en el desempeño laboral, situación que antes no se había tenido en cuenta, y que era causa generadora de malestar en la sociedad industrial (Aktouf, 2009; Marín-Idárraga, 2006; Mayo, 1977).

Posteriormente, se desarrollan una serie de postulados administrativos que abogan por posturas más eclécticas o abarcadoras; tal es el caso de la escuela neoclásica, que en medio de una jungla epistemológica emerge con presunciones de universalidad y objetividad, fundadas en concepciones científicas (Koontz, 1999). De la misma manera, se desarrollaron otros enfoques como el de las ciencias del comportamiento (basado en el enfoque humanista), que exploraban las necesidades humanas desde los conceptos de liderazgo, motivación, entra otros (Herzberg, Mausner, \& Snyderman, 2011; Maslow,1991; McGregor, 1994). También, se gestó el movimiento estructuralista como resultado de la llegada tardía de los textos de Weber, promoviendo la conciliación entre los clásicos y los humanistas (Blau, 1964; Etzioni, 1975); considerada una teoría transitoria hacia la concepción sistémica en la administración.

En síntesis, la evolución del sustrato teórico de la administración se dirigió hacia interpretaciones que toman en consideración el entorno organizacional y la tecnología, entre otras características, las cuales fueron el fundamento de la escuela contingencial (Burns \& Stalker, 1961). En este orden de ideas, la interrelación con el ambiente mostró la necesidad de explicar a las organizaciones como sistemas abiertos; cuyos procesos son complejos (Katz \& Kahn, 1966), eso significa que tienen capacidad de influir y de ser influidos por su medio; ellas conglomeran un conjunto de personas que ponen a disposición sus capacidades como el capital intelectual para la consecución de un objetivo común (Aktouf, 2009; Morgan, 1998).

Es evidente como, con el transcurrir del siglo XX y el desarrollo de las ciencias de la gestión, el sustrato mecanicista empieza a cambiar paulatinamente; sin embargo, hay que anotar que se mantienen en vigencia algunos de los planteamientos esbozados, provenientes del contexto económico preponderante (Aktouf, 2009; Chanlat, 2011; Gallardo, 2002; Merlano, 1996; Montoya \& Montoya, 2002; Morgan, 1998). Esta inhabilidad para desprenderse de la lógica de la utilidad o del beneficio financiero, ha planteado la concepción de que las teorías organizacionales se han desenvuelto, como discursos ideológicos que se manifiestan en oleadas normativaso racionales según la necesidad del contexto económico (Barley \& Kunda, 1992).

En consecuencia, las organizaciones se muestran así mismas como instrumentos del sistema económico, impactando en la sociedad y el ambiente, desde la era industrial (Barley \& Kunda, 1992); efectos que, en la actualidad, a la luz de los desarrollos en materia ecológica, cobran mayor relevancia; y más aún en un contextocaracterizadopor laglobalización, cuyo fenómeno se vislumbra desde una concepción de interconectividad compleja, constituido por variables políticas, económicas, ambientales y culturales (Acosta \& González, 2007). De allí que al problema ambiental que aqueja a la sociedad contemporánea, se le suelen atribuir raíces económicas que provienen de la concepción ilimitada de los recursos 
naturales que se planteó en la economía clásica (Naredo, 2006). Estas, y otras cuestiones, muestran los impases de la modernidad, y la necesidad de re-orientar el comportamiento organizacional global.

En este orden de ideas, la necesidad de comprender a las organizaciones, sus fenómenos y contextos de forma más acertada a la realidad social y ambiental es imperativa. Por lo cual, la visión radicalmente amplia que ofrece la termodinámica del no-equilibrio en el marco de las ciencias de la complejidad, juega un papel trascendental. En primer lugar, porque las organizaciones contemporáneas son, en esencia, sistemas que no están en equilibrio; los cuales viven procesos de adaptación y auto-organización; además están compuestos por seres humanos, cuyos aspectos biológicos, sociales, psicológicos, físicos, entre otros; constituyen propiamente, sistemas complejos (Gallardo, 2002; Herrero, 2008; Morúa, 2015; Prigogine \& Stengers, 1994; Pulgarín, 2013; Tovar, 2009).

La dinámica organizacional, caracterizada por su comportamiento como organismo vivo está sometida a cambios, fluctuaciones, inestabilidades que se presentan en su ambiente interno y externo, y que generan desorden o desequilibrios. Pero por su misma naturaleza, es capaz de evolucionar y emerger a un estado de orden dinámico. Esta forma de entender a las organizaciones, rompe el principio de causalidad en ellas, tal que, las causas y los efectos dejarían de ser proporcionales y cobraría importancia la sensibilidad de las condiciones iniciales de un sistema; así como, la emergencia de cambios súbitos, y la interrelación entre las estructuras poli-niveladas que constituyen a las organizaciones y de las cuales estas hacen parte (Capra, 1992; Villalba, 2012; Yoris, 2017).
En suma, los sistemas organizacionales vistos desde la termodinámica del noequilibrio, o más bien, como estructuras disipativas evidencian la necesidad de reinterpretar el sustrato teórico bajo el cual se fundamenta la concepción organizacional. De esta manera, dichos sistemas se pueden apreciar como conglomerados o sistemas sociales que afectan al ambiente, de tal forma que existiese una preocupación por los procesos internos y los impactos externos que ocasionan. Por tanto, y a causa de la naturaleza dinámica, inestable, impredecible y adaptativa de las estructuras disipativas (como organizaciones), afirma Prigogine emergería en los estados lejanos al equilibrio comportamientos colectivos, un régimen de actividad coherente, que se imposibilita desde estados de equilibrio (Prigogine, 1996; Villalba, 2012).

\section{CONCLUSIÓN}

El predominio del paradigma regente impuesto desde la modernidad ha alcanzado sus límites. No obstante, las raíces de la transformación que se gestó durante todo el siglo XX, y que desembocó en el cambio de paradigma, tuvo sus antecedentes en los inicios de la termodinámica clásica, cuyos aportes se destacaron por ser los primeros en no pertenecer al edificio de la mecánica newtoniana. Siguiendo la línea de avance de la termodinámica, se hallan las concepciones de energía y entropía, importantes en el estudio de los sistemas termodinámicos, o en equilibrio; y más adelante, en los del no-equilibrio. La concepción de no-linealidad desarrollada en el transcurso del siglo XIX, fue precursora para los estudios de Prigogine sobre la termodinámica en estados alejados del equilibrio.

Los descubrimientos del químico Belga contribuyeron a consolidar, junto con otros 
adelantos, las ciencias de la complejidad, aquellas caracterizadas por la incertidumbre, contradicción, totalidad. Estos aportes han sido poco a poco extrapolados al ámbito de la gestión administrativa, donde se empieza a generar la comprensión de las organizaciones como sistemas alejados del equilibrio, o en términos del nobel de química, estructuras disipativas. Esta nueva concepción, que promulga la dinámica e inestabilidad como características inherentes a los sistemas complejos, así como su invariante relación con el medio ambiente, la sociedad y los procesos caracterizados por exhibir vida, como los humanos, se espera logren contribuir a la solución de las problemáticas que aquejan a las organizaciones enmarcadas en el contexto contemporáneo.

Sin embargo, es fundamental la emancipación de la lógica utilitarista, para avanzar en la comprensión de las organizaciones como estructuras disipativas. Este importante campo de las ciencias contem- poráneas se muestra como una forma más acertada de comprensión de la realidad, siendo la vida el más claro ejemplo de complejidad, al igual que los ecosistemas son el mayor modelo de sostenibilidad. No obstante, es importante tener presente que el modelo social construido a partir de la modernidad no es coherente con los principios que soportan el desarrollo de la naturaleza, construyendo un mundo antinatural que responde por sobre todas las cosas a la lógica de la maximización, cosmovisión que ha afectado seriamente a la biosfera, al hombre mismo y a toda la vida sobre el planeta, al concebir un mundo desconectado y en función de su utilidad y satisfacción de las necesidades humanas, que bajo los planteamientos neoclásicos son ilimitadas. Esta carrera hacia la destrucción hace urgente la necesidad de nuevos referentes para el abordaje de la realidad organizacional, teniendo presente y siendo conscientes que se puede caer en una mera refinación de la teoría administrativa, si no es superada la ideología de la economía neoclásica.

\section{REFERENCIAS BIBLIOGRÁFICAS}

Acosta, O., \& González, J. (2007). Globalización: una aproximación desde la evolución biológica y los sistemas complejos auto-organizativos. Análisis Político 20 (61), 101-121.

Aktouf, O. (2009). La adminsitración: entre tradición y renovación. Cali: Artes gráficas del Valle.

Álvarez, A. (2010). Frederick Winslow Taylor y la administración científica: contexto, realidad y mitos. Revista Gestión y estrategia (38), 17-30.

Barabasi, A. (2002). Linked: How everything is connected to everything else and what it means. Plume editors.

Barley, S. R., \& Kunda, G. (1992). Design and devotion: surges of rational and normative ideologies of control in managerial discourse. Administrative Science Quarterly ASQ 37, 363-399.

Bertalanffy, L. V. (1989). Teoría general de los sistemas. Fundamento, desarrollo, aplicaciones. Mexico: Fondo de Cultura Económica.

Blau, P. (1964). Exchange and power in social life. Transaction Publishers.

Bohr, N. (1934). Atomic physics and the description of nature.Cambridge University.

Bunge, M. (2004). Emergencia y convergencia. Novedad cualitativa y undad del conocimiento. Barcelona: Gedisa. 
Burns, T., \& Stalker, G. (1961). The management of innovation.

Capra, F. (1992). El punto crucial. Buenos Aires: Editorial Troquel S.A.

Capra, F. (1998). La trama de la vida: una nueva perspectiva de los sistemas vivos.Barcelona: Anagrama.

Carnot, S. (1897). Reflections on the motive power of heat. Nueva York: John Wiley \& Sons.

Chanlat, A. (2011). Las ciencias de la vida y la gestión administrativa. Cuadernos de Administración 10 (14), 26-34.

Durán, R. (2016). Vida y materia: Bergson y la termodinámica clásica. Veritas (34), 75-91.

Einstein, A., \& Infeld, L. (1986). La evolución de la física . Barcelona: Salvat Editores S.A.

Elizalde, O. A. (2013). Aproximación a las ciencias de la complejidad. Revista Universidad de la Salle (61), 45-66.

Etzioni, A. (1975). Comparative Analysis of Complex Organizations. Rev. Simon and Schuster.

Fayol, H. (1917). Administration Industrielle et Générale. Paris: H. Dunod et E. Pinat, Éditeurs.

Fromm, E. (1964). Psicoanalisis de la sociedad contemporánea. México-Buenos Aires: Fondo de cultura económica.

Gallardo, A. (2002). La era de la incertidumbre, la organización y la teoría del caos. Administración y Organizaciones, 63-76.

García-Olivares, A. (1999). La evolución de la complejidad. Empiria. Revista de metodología de ciencias sociales (2), 93-128.

Gell-Mann, M. (1998). El quark y el jaguar. Aventuras en lo simple y lo complejo. Barcelona: Tusquets Editores.

Georgescu-Roegen, N. (1977). ¿Qué puede enseñar a los economistas la termodinámica y la biología? Atlantic Economic Journal 5, 13-21.

Georgescu-Roegen, N. (1996). La ley de la entropía y el proceso económico.Madrid: Fundación Argentaria.

Giorgine, B., \& Turchetti, G. (2006). From Newton-Boltzmann paradigms to complexity: a bridge to bio-systems. The Science of Complexity: Chimera or Reality, Milan Research for Industrial and Applied Mathematics , 1-16.

Gómez, D. (2014). Apuntes desde la ciencia económica ¿el sujeto racional o el sujeto complejo? Clío América 8 (15), 83-89.

Goméz-Cruz, N. A., \& Maldonado, C. E. (2011). El mundo de las ciencias de la complejidad. Bogotá: Universidad del Rosario.

González, J. (2010). Factor humano en el trabajo desde la perspectiva de la escuela de las relaciones humanas: reflexión para la Región. Revista Páginas (86), 67-78.

Heisenberg, W. (1959). Física y filosofía. Buenos Aires: Editorial La Isla. 
Heisenberg, W. (1985). La imagen de la naturaleza en la fisica actual. Barcelona: Ediciones Orbis S. A.

Herrero, L. (2008). Del mecanicismo a la complejidad en la biología. Revista de biología tropical 56 (1), 399-407.

Herzberg, F., Mausner, B., \& Snyderman, B. (2011). The motivation to work. Transaction Publishers.

Katz, D., \& Kahn, R. (1966). The social psychology of organizations. New york: Wiley.

Kondepudi, D., \& Prigogine, I. (2014). Modern thermodynamics: from heat engines to dissipative structures.John Miley \& Sons.

Koontz, H. (1999). La jungla de la teoría administrativa. Revista Contaduría y Administración (193), 37-52.

Koyré, A. (1979). Del mundo cerrado al universo infinito. Madrid: Siglo XXI.

Koyré, A. (2008). Los albores de la ciencia clásica. ContactoS 69, 43-48.

Kuhn, T. (1962). The structure of scientific revolutions. University of Chicago Press.

Kuhn, T. (1978). La revolución copernicana. La astronomía planetaria en el desarrollo del pensamiento occidental. Barcelona : Editorial Ariel .

La Mettrie, J. O. (1987). El hombre máquina. Editorial Alhambra.

Le Moigne, J. L. (1997). La incoherencia epistemologica de las ciencias de la gestión. Cuadernos de economía 16 (26), 163-185.

López, S. (2005). Lo epistemologico para hablar de adminsitración. Revista Páginas (73), 3-12.

Lorenz, E. (1963). Deterministic nonperiodic flow. Journal of the atmospheric sciences 20 (2), 130-141.

Lovelock, J., \& Margulis, L. (1996). The Gaia Hypothesis.

Maldonado, C. E. (2011). Termodinámica y complejidad: una introducción para las ciencias sociales y humanas. Bogotá: Ediciones desde abajo.

Mandelbrot, B. (1982). The fractal geometry of nature.

Marin, C. (2014). Entropía. Un cadaver exquisito. AusArt Journal for Research in Art 2 (1), 223-233.

Marín-Idárraga, D. A. (2006). El sujeto humano en la administración: una mirada crítica. Cuadernos de Administración 19 (32), 135-156.

Marín-Idárraga, D. A. (2012). Consideraciones epistemológicas en torno al carácter científico de la administración. Innovar 22 (46), 39-52.

Martínez. (1996). Termodinámica y epistemología. Revista mexicana de física 42 (3), 488-497.

Martínez, F., Ortiz, E., González, A., \& Brito, H. (2009). Antecedentes, iniciadores y fundamentos de los estudios en complejidad. Quórum Académico 6 (1), 79-120.

Maslow, A. (1991). Motivación y personalidad. Ediciones Díaz de Santos. 
Maturana, H., \& Varela, F. (1990). El árbol del conocimiento: las bases biológicas del conocimiento humano. Madrid: Debate.

Mayo, E. (1977). Problemas sociales de una civilización industrial. Buenos Aires: Ediciones nueva visión.

McGregor, D. (1994). El lado humano de las organziaciones. Santafé de Bogotá: McGraw-Hill.

Merlano, A. (1996). Paradigmas emergentes de la ciencia y su impacto en la adminsitración del siglo XXI. Cuadernos de Administración (23), 21-38

Montoya, I. A., \& Montoya, A. (2002). El nuevo paradigma de las ciencias y la teoría de gestión. Innovar: revista de ciencias administrativas y sociales, 17-34.

Mora, J., \& Pérez, C. (2006). Termodinámica y evolución biológica, ¿amigos o enemigos? Universidad Autónoma de Baja Californa, 40-44.

Morgan, G. (1998). Imagenes de la organización. México D. F.: Alfaomega Grupo Editor S. A.

Morúa, J. (2015). Entropía y dinámica, ¿qué nuevas contribuciones para las ciencias de la gestión? UPIICSA investigación interdisciplinaria 1 (1), 26-40.

Mumford, L. (1998). Técnica y civilización. Alianza Editorial.

Najmanovich, D. (2005). La metamorfosis de la ciencia. Suplemento futuro.

Naredo, J. M. (2003). La economía en evolución: historia y perspectivas de las categorías básicas del pensamiento económico. Madrid: Siglo XXI de España Editores S. A.

Naredo, J. M. (2006). Raíces económicas del deterioro ecológico y social. Más allá de los dogmas. Madrid: Siglo XXI de España Editores S. A.

Pérez, J. R. (2007). La Ciencia Europea antes de la Gran Guerra. La termodinámica de Carnot a Clausius (págs. 1-21). Orotava y Las Palmas de Gran Canaria: Fundación Canaria Orotava.

Prigogine, I. (1980). From being to becoming. Time and complexity in the physical sciences.New York: W. H. Freeman and Company.

Prigogine, I. (1993). ¿Tan solo una ilusión? . Tusquets Editores S. A.

Prigogine, I. (1996). El fin de las certidumbres.

Prigogine, I. (1997). El desorden creador. Tomado del original en francés:" Le désordre créateur". Tribune libre à I. Prigogine, Institut du management d'EDF/GDF.

Prigogine, I. (2006). El nacimiento del tiempo. Buenos Aires: Tusquets Editores S. A.

Prigogine, I., \& Stengers, I. (1984). Order Out of Chaos. New York: Bantam books.

Prigogine, I., \& Stengers, I. (1991). Entre el tiempo y la eternidad. Buenos Aires: Alianza Editorial.

Prigogine, I., \& Stengers, I. (1994). La nueva alianza: metamorfosis de la ciencia. Madrid: Alianza Editorial.

Pulgarín, S. A. (2013). De las organizaciones: reflexiones y aproximaciones desde la biología y la complejidad. Criterio Libre (18), 195-216. 
Sandino, A. F., \& Montiel, L. (2012). La termodinámica como origen de la revolución industrial del siglo XVIII. Lat. Am. J. Phys. Educ 6 (4), 652-654.

Schrödinger, E. (1997). La naturaleza y los griegos. Isytax.

Schrödinger, E. (2005). ¿Qué es la vida? Salamanca.

Strogatz, S. (2003). Sync: How order emerges from chaos in universe, nature and daily life. Hyperion.

Taylor, F. W. (1919). The principles of scientific management. Harper \& brothers.

Thom, R. (1989). Structural stability and morphogenesis.

Torreblanca, J. (2013). Origen de la termodinámica, el enigma de la energía. Remetallica (3), 22-25.

Tovar, L. A. (2009). Evolución de la teoría de la organización. Universidad \& Emrpesa 17, 11-32.

Turegano, J. A., \& Valero, A. (1982). Los origenes de la termodinámica y su paralelismo actual. Actas II Congreso de la Sociedad Española de Historia de las Ciencias (págs. 383-388). Universidad de Zaragosa.

Villalba, M. E. (2012). Gestión con base en las ciencias de la complejidad: las organizaciones como estructuras disipativas. Universidad \& Empresa (22), 11-42.

Watts, D. (2004). Six degrees: the science of a connected age.Norton \& Company.

Yoris, O. (2017). Gerencia del No Equilibrio: Tendencia Disruptiva en las Organizaciones del siglo XXI. TELOS. Revista de Estudios Interdisciplinarios en Ciencias Sociales 19 (2), 331-342.

Zadeh, L. (1965). Fuzzy sets. Information and control 8 (3), 338-353.

\section{Para citaciones:}

Carbal-Herrera, A., Guevara-Huertas, Y. M., \& Ochoa-Uparela, J. Y. (2017). Las Organizaciones desde las ciencias de la Complejidad: Una aproximación desde la termodinamica del no-equilibrio. Panorama Económico, 25, 1, pp. 75-94.

\section{AUTORES}

\section{Adolfo Carbal-Herrera}

Profesor Asistente e Investigador del Programa de Contaduría Pública de la Facultad de Ciencias Económicas de la Universidad de Cartagena (Colombia). Actualmente Doctorante en Administración de la Universidad de Medellín (Colombia) y director del Grupo de Investigación GIDEA - Universidad de Cartagena.

\section{Yury Marcela Guevara-Huertas}

Integrante del Semillero de Investigación de Estudios Medioambientales SIDEMA de la Universidad de Cartagena.

Jaime Yasser Ochoa-Uparela

Integrante del Semillero de Investigación de Estudios Medioambientales SIDEMA de la Universidad de Cartagena. 


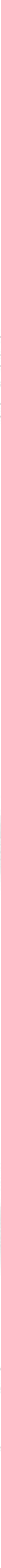

